\title{
Philosophiques
}

\section{Oeuvres de Charles De Koninck, tome I, Philosophie de la nature et des sciences, vol. 1, Québec, PUL, 2009, 475 p.}

\section{Jean-Claude Simard}

Volume 38, numéro 1, printemps 2011

URI : https://id.erudit.org/iderudit/1005739ar

DOI : https://doi.org/10.7202/1005739ar

Aller au sommaire du numéro

Éditeur(s)

Société de philosophie du Québec

ISSN

0316-2923 (imprimé)

1492-1391 (numérique)

Découvrir la revue

Citer ce compte rendu

Simard, J.-C. (2011). Compte rendu de [Oeuvres de Charles De Koninck, tome I, Philosophie de la nature et des sciences, vol. 1, Québec, PUL, 2009, 475 p.]

Philosophiques, 38(1), 365-372. https://doi.org/10.7202/1005739ar

Ce document est protégé par la loi sur le droit d'auteur. L'utilisation des services d'Érudit (y compris la reproduction) est assujettie à sa politique d'utilisation que vous pouvez consulter en ligne.

https://apropos.erudit.org/fr/usagers/politique-dutilisation/ 
gramme deleuzien, qui préconisait l'expérimentation ouverte et récusait tout monopole du sens. Après tout, Dionysos a bien été démembré, et la dispersion contrôlée a aussi son charme.

JEAN-CLAUDE SIMARD

Collège de Rimouski

\section{Euvres de Charles De Koninck, tome I, Philosophie de la nature et des sciences, vol. 1, Québec, PUL, 2009, 475 P.}

Cette publication rend disponible une œuvre majeure de l'histoire de la pensée québécoise au $\mathrm{xx}^{\mathrm{e}}$ siècle. En effet, en plus d'être le père de plusieurs chercheurs universitaires reconnus et d'avoir laissé son nom à un pavillon de l'Université Laval, Charles De Koninck fut un animateur important de la vie philosophique et intellectuelle du Québec. Les PUL comptent publier ses œuvres complètes, dont voici l'ouvrage inaugural.

On a ordonné les textes selon une logique thématique, et c'était justice d'ouvrir le tout par la philosophie des sciences et de la nature, champ de prédilection de l'auteur. Rappelons-le, c'est dans ce domaine qu'il enseigna; d'ailleurs, sa thèse de doctorat en philosophie (Louvain, 1934) portait sur les travaux de l'astrophysicien Eddington.

Ce volume regroupe neuf textes, échelonnés de 1936 à 1960. On y trouve d'abord deux ouvrages: Le cosmos (1936) et L'univers creux (1960, inédit en français), ainsi que l' 'Introduction à l'étude de l'âme» de 1947, l'importante préface au Précis de psychologie thomiste de l'abbé Stanislas Cantin. On y a ajouté six articles: "Le problème de l'indéterminisme» (1937), «Mathématiques et philosophie» (1940), "Les sciences expérimentales sont-elles distinctes de la philosophie de la nature ?» (1947), «Un paradoxe du devenir par contradiction" (1954), ainsi que "Le problème de l'évolution" et "La responsabilité morale du scientifique" (inédit), tous deux de 1950. L'ensemble est précédé d'un avant-propos de Thomas De Koninck, ainsi que d'une présentation d'Yves Larochelle, maître d'œuvre de ce premier volume, lequel a aussi traduit les deux textes anglais. Un index onomastique et une bibliographie des œuvres du philosophe consacrées aux sciences viennent clore le tout.

Moins étudié sans doute que Maritain et Gilson, De Koninck constitue pourtant un relais cardinal de la première moitié du dernier siècle. Évidemment, la facture de ces textes témoigne d'une époque révolue; l'auteur y conjugue en effet trois niveaux distincts: les développements scientifiques, l'hylémorphisme aristotélicien et la théologie catholique. Comme le dit Larochelle dans sa présentation, ce style trouve encore des adeptes à l'Uni-

* Je remercie l'évaluateur anonyme de Philosophiques pour ses commentaires sur la version initiale de cette recension. 
versité Laval, mais il constitue "une exception québécoise, voire continentale» (XXIV).

Cela dit, l'intérêt de ces textes se trouve ailleurs. D'une part, ils incitent à nuancer l'idée habituelle de Grande Noirceur. Au Québec, les années 30 constituent en effet une période d'ouverture internationale. À l'Université Laval, c'est la fondation de la Faculté des sciences (1937) et du Département de physique (1939). L'arrivée du philosophe d'origine belge s'inscrit dans le cadre de cette fenêtre ouverte sur le monde, que l'emprise duplessiste va ensuite chercher à refermer. D'ailleurs, De Koninck est sans doute le premier penseur francophone canadien (du moins d'adoption) qui ait ainsi rayonné hors de nos frontières. D'autre part, on prétend souvent que l'idée d'évolution biologique est alors rejetée au Québec, sauf par quelques esprits isolés, tel le frère Marie-Victorin. Il faut aussi tempérer cette perception. De Koninck est non seulement familier avec les travaux de Lamarck, Darwin, de Vries et von Bertalanffy, mais il soutient que «le philosophe qui nie la possibilité [...] d'une théorie évolutionniste nie l'essence même de la méthode scientifique» (84).

Abordons à présent le contenu lui-même. Le cosmos, texte le plus développé du recueil, veut offrir une philosophie de la nature en phase avec le siècle. La première partie, "Le point de vue scientifique " (3-25), installe le décor en rappelant quelques acquis majeurs de l'astrophysique, de la cosmologie et de l'évolutionnisme modernes. Plus étoffés, les quatorze chapitres de la deuxième partie ( "Le point de vue philosophique ", 26-117) dévoilent l'arrière-scène. On sait que, depuis Galilée, la méthode scientifique exclut les causes finales et la recherche de l'essence. Stigmatisant cette approche, De Koninck entend démontrer que, pour sa part, le philosophe ne saurait s'en passer. Selon lui, le but de l'évolution terrestre était de produire un corps digne d'abriter une âme supérieure, celui qu'appelle justement l'esprit humain (65-81). Cet anthropocentrisme clairement assumé affirme «l'impossibilité absolue d'un cosmos qui ne soit essentiellement ordonné à l'homme» $(86$, n. 76$)$, de sorte que l'histoire de la vie, voire de l'univers même, aurait tendu vers notre espèce ${ }^{1}$. Quoi qu'on pense de ces thèses, il faut en saluer l'envergure: incarnant l'homme dans la matière et dévoilant une causalité immanente au Cosmos, elles font paraître bien puéril l'occasionnalisme des partisans du créationnisme ou du Dessein intelligent. Les quatre chapitres de la dernière partie, «Le point de vue théologique " (118$130)$, viennent coiffer ce finalisme en présentant le metteur en scène. De Koninck y propose une lecture pour le moins audacieuse de la cosmologie.

1. Sans doute parce que ses écrits autres que scientifiques étaient alors interdits de publication au Québec et que, pour le lire, on devait consulter des polycopies circulant sous le manteau, Teilhard n'est nulle part mentionné par De Koninck. Pourtant, plusieurs passages du texte, par exemple la section 12 , p. 85-97, témoignent clairement de son influence et, à travers lui, de celle de Bergson, son inspirateur. 
Il enjambe d'abord la théologie naturelle, selon lui limitée à l'intelligence humaine, pour faire appel à ce qu'il appelle la «Théologie sacrée». Voyant en l'univers un vestige de la divinité, et en l'homme son image atténuée, cette voie interprétative prétend décrypter l'histoire du cosmos à la lumière des niveaux de la Trinité chrétienne... Malheureusement, cette troisième partie étant inachevée, nous ne connaîtrons jamais le détail de cette surprenante procession ontologique, déjà esquissée dans la deuxième partie de l'ouvrage (section 13, 98-109).

Mariant culture scientifique, philosophie scolastique et théologie, cet ouvrage de 1936 donne le ton du volume tout entier. De Koninck habille en effet les derniers résultats de la science de vêtements médiévaux, une opération qui laisse perplexe. Elle évoque ces films d'époque où des chevaliers en armure, maniant la lance et chevauchant leurs destriers, expriment, dans un langage contemporain, les préoccupations de l'heure. Le tout exhale un parfum suranné, comme ces pièces longtemps closes dont l'odeur, éveillant maints souvenirs enfouis, ébranle, de proche en proche, les diverses couches de la mémoire.

Le second texte, «Le problème de l'indéterminisme» (297-347), s'attaque aux résultats troublants de la physique quantique. De Koninck veut s'appuyer "sur la lettre de saint Thomas et de ses grands commentateurs" pour examiner «ces problèmes [...] nouveaux que [les] maîtres du passé ne pouvaient soupçonner» (298). Un rappel du déterminisme en mécanique classique l'amène à expliquer la teneur des célèbres relations d'incertitude dont, curieusement, il ne crédite pas Heisenberg. Il rejette ensuite le principe de causalité absolu pour défendre, avec Eddington, l'interprétation standard de la mécanique quantique. La deuxième partie de l'article aborde l'indéterminisme métaphysique. De Koninck y examine longuement la célèbre question aristotélicienne des futurs contingents (De interpretatione, chap. IX), ainsi que celle du hasard et de la nécessité (Phys. II, chap. 4-6). On le sait, ces questions difficiles avaient jeté les penseurs du Moyen-Âge, qui tenaient pour acquise l'existence d'un Être omniscient, dans des abîmes de perplexité. Après une discussion subtile, fidèle au thomisme, De Koninck conclut qu'il "faut distinguer [...] la contingence de la nature de la contingence du hasard» (332). Cela admis, on doit reconnaître "un certain jeu dans les limites de la nature» (338), auquel cas l'indéterminisme quantique devient tout à fait acceptable. Enfin, une brève troisième partie (341-347), de nature plus théologique, conclut en rappelant l'indétermination principielle de toute matière, nécessaire pour accueillir une réalité spirituelle telle l'âme humaine.

Cette «philosophia perennis» (235), celle du Prince des philosophes amendée par le Docteur Angélique, imprègne aussi les essais sur la biologie. "Le problème de l'évolution ", un texte qui préfaçait le vol. II de La doctrine de l'évolution, de l'abbé Louis-Eugène Otis (Montréal, Fides), se contente d'en présenter le principe. Quoique très ambigu, nous le verrons, «Un para- 
doxe du devenir par contradiction" (231-284) constitue par contre un texte de fond, sur lequel il convient de s'arrêter un moment. De Koninck y défend la notion de devenir, un des trois types de mouvement selon Aristote. Il critique d'abord sévèrement l'idée de changement comme processus de résolution des contradictoires, un concept inspiré de Hegel et repris par les marxistes. (Notons en passant que citer de copieux extraits de Lénine, comme il le fait ici, témoigne d'une ouverture d'esprit peu commune à l'époque.) De Koninck table plutôt sur le devenir comme processus de résolution des contraires, du moins pour l'acception habituelle du changement. Cependant, il adopte in fine la logique des contradictoires pour traiter l'épineux problème de la transsubstantiation, dont il propose une interprétation philosophique osée. À cette fin, il forge le concept de «devenir absolu » et entend réhabiliter la notion aristotélicienne de matière première (263-265).

Le lecteur qui aura suivi attentivement cette démonstration serrée courant sur cinquante pages sera évidemment déçu. Au terme de ces savants développements sur le temps, l'instant, le devenir, l'être et le non-être, il aurait souhaité quelque habile application à la question de l'évolution biologique ou un éclairage inédit sur de difficiles problèmes actuels. Au lieu de quoi il constate que ces trésors d'érudition et de finesse visaient l'élucidation de la transsubstantiation, un problème théologique qui, fût-il crucial pour le catholique, porte bien peu à conséquence pour le philosophe. Philosophia ancilla theologice!

"Mathématiques et philosophie» (133-140) constitue un plaidoyer en faveur des mathématiques, "la plus humaine des sciences». Selon De Koninck, la recherche de sagesse ne saurait en effet se priver d'une "philosophie des mathématiques", sous peine de s'exercer dans le vide. Dans ce trop bref texte, il situe les mathématiques par rapport à divers domaines traditionnels, comme la philosophie de la nature ou la métaphysique. Retenons-en cette louable injonction: "On ne demande pas tant au philosophe de juger - les jugements n'ont pas fait défaut; on demande qu'avant de juger il sache de quoi il s'agit» (136). Cela dit, en raison de leur concision, certains passages sont malaisés à interpréter. À preuve: "Les philosophes des mathématiques contemporains professent soit un mathématisme de couleur platonicienne, soit un relativisme sophistique» (137). De Koninck évoque sans doute ici la célèbre question des fondements. Le "mathématisme de couleur platonicienne» désigne probablement les diverses formes de réalisme. Quant au "relativisme sophistique », prétend-il englober le formalisme (Hilbert), le logicisme (Russell) et l'intuitionnisme (Brouwer et Heyting)? Si oui, un tel laminage des positions philosophiques sur le statut des mathématiques est pour le moins extravagant. À moins que l'auteur ne s'en prenne aux travaux des philosophes professionnels, excluant les mathématiciens philosophes? Mais dans ce cas, qui vise-t-il, au juste? Malheureusement, on ne connaîtra sans doute jamais la réponse à ces questions... 
«Les sciences expérimentales sont-elles distinctes de la philosophie de la nature?» (141-152) dissocie les sciences de la nature, attachées à des hypothèses provisoires, de la philosophie de la nature, moins assujettie au progrès des connaissances. De Koninck y défend en outre Aristote contre les interprétations hâtives en montrant qu'il savait parfaitement départager ces deux ordres de connaissance, qu'il fut le premier à caractériser clairement. Quant au texte suivant, «La responsabilité morale du scientifique» (351358), il rappelle que le progrès des connaissances n'entraîne pas forcément un progrès moral, et que la vertu aristotélicienne de prudence demeure cardinale pour tous, qu'on soit ou non un scientifique.

La longue "Introduction à l'étude de l'âme» (155-231) présente et justifie les bases de la psychologie et de la gnoséologie thomistes. Fort de ce conceptualisme, De Koninck en profite pour égratigner au passage les points de départ philosophiques de Hegel (179) ou de Husserl, Bergson, Descartes et saint Augustin (165sq.). Mais, parce qu'elle disjoint essences et matière, ses critiques les plus sévères s'adressent à la méthode platonicienne. Car le "mouvement de concrétion" doit expliquer l'âme de manière rigoureuse, donc par les causes, sans pour autant négliger l'être singulier. C'est ce double cheminement qui, dans sa tension, crée la connaissance véritable. L'on parvient ainsi à la définition de l'âme, objet de la "psychologie rationnelle»: "l'acte premier d'un corps naturel muni d'instruments» (195). De Koninck lie ensuite l'âme à l'intelligence, par le biais des facultés sensibles: "N'étant pas l'acte d'un organe, l'intelligence a sa concrétion dans l'âme» (201). De telles positions feront sourciller quiconque est familier avec la neurologie, mais, malheureusement, l'auteur demeure ici totalement muet sur le rôle potentiel du cerveau. La dernière section du texte (206-230) propose ce qu'on pourrait appeler une forme d'opérationnalisme, une position commode pour qui veut limiter la portée des résultats scientifiques, et préserver l'espace propre à la philosophie, la "science spéculative» (226).

L'univers creux, enfin, constitue une exception notable dans cet ensemble. Seul ouvrage achevé et écrit polémique, ce texte est selon nous le plus surprenant du volume. La première section, "Le monde des constructions symboliques, ou deux c'est un deux fois " (365-396), se penche sur le statut du nombre: l'auteur refuse d'y voir un simple symbole, par exemple une classe de classes comme Russell, bien qu'il reconnaisse là une optique utile pour le calcul. S'opposant à l'approche analytique, il défend plutôt le point de vue aristotélicien de l'abstraction à partir du concret: le "déni des termes analogiques, utilisés comme moyens d'expression intelligibles, signifie l'impossibilité de toute philosophie » $(378, \mathrm{n} .17)$. Il rend «creuses ${ }^{2}$ », c'est-à-dire purement opérationnelles et vides de sens, les mathématiques. Il faut en conséquence écarter la conception du nombre comme fiction com-

2. Comme le rappelle le traducteur, le terme anglais utilisé, hollow, fait directement référence au titre même de l'ouvrage. 


\section{0 • Philosophiques / Printemps 2011}

mode, sans pour autant retomber dans les apories médiévales liées aux Universaux. De même, il convient de rejeter la notion cantorienne d'infini en acte, pour s'en tenir à l'infini en puissance d'Aristote ${ }^{3}$. La deuxième section, "La construction mentale et le test de l'expérience» (397-427), interroge le caractère vide des mathématiques, lorsque coupées de l'expérience. Pour étayer l'analyse, l'auteur examine des cas précis: l'utilisation de la Théorie algébrique des groupes, socle, on le sait, du futur modèle standard en physique des particules, les notions d'esprit, de mouvement, d'individu humain, etc. Il en profite pour défendre, contre Russell, la conception aristotélicienne du temps et de la substance, car les définitions opérationnelles de ces notions, les «nombres-mesures» de la science, n'en épuisent pas la signification. Quant à la Nature en soi, elle ne dévoilera sans doute jamais ses secrets, mais il faut tout de même l'interroger, car, dit-il dans une formule sévère, pratiquée par des hommes vides (hollow men), la philosophie contemporaine rend l'univers creux (hollow) lorsqu'elle persiste à éluder la question du sens $(427)^{4}$. Après la physique mathématique, la troisième et dernière partie (428-457) aborde, à l'enseigne d'un titre percutant («Le monde sans vie de la biologie»), l'univers du vivant. De Koninck y reproche à la science de traiter la notion de vie de l'extérieur, excluant toute définition véritable de ce terme, plutôt clair pour le sens commun. Le problème tiendrait au physicalisme cartésien, une approche mécaniste pourtant abandonnée, même en physique. Il en va de même pour le phénomène de l'évolution, ou pour des termes comme organe, organisme, etc. Considérons la main humaine: peut-on la comprendre sans faire appel à la finalité (442-444) ? De Koninck plaide donc en faveur de l'explication téléologique, tout en refusant l'étiquette anthropomorphiste. En d'autres termes, il voudrait que la biologie délaisse les modèles hérités de la physique, pour revenir à l'analogie aristotélicienne entre l'art et la nature (453 sq.). Ainsi, elle aussi serait moins vide (hollow). Dans un bref épilogue intitulé "Règlement de comptes avec les ordinateurs» (458-467), l'auteur s'attaque à l'intelligence artificielle, une forme de scientisme comparable, selon lui, au réductionnisme mathématique, physique ou biologique. S'appuyant sur une citation de Turing, il ridiculise ceux qui prétendent abolir la différence entre calcul mécanique et pensée humaine.

Au vu de ce premier volume, que penser du travail de Charles De Koninck ? Il mobilise le vocabulaire habituel de l'aristotélico-thomiste: puissance et acte, entéléchie, matière et forme, âme végétative, etc. Il crée d'ailleurs parfois des néologismes insolites, calqués sur le latin médiéval:

3. Signalons à ce propos un impair (de l'original ?): aleph-zéro, le premier cardinal transfini de Cantor, est symbolisé par ? $?_{0}$, et non par $\infty_{0}$ (p. 392).

4. Bien que la chose ne soit nulle part mentionnée dans le texte, on peut supposer que la métaphore de l'univers creux ainsi que la référence aux «hommes vides» est directement inspirée du poème de T. S. Eliot, Hollow Men (1925). 
puissance cognoscitive (204), sens entitatif (207), détermination quidditative, etc. Cela dit, il connaît bien les philosophes d'allégeance anglo-saxonne, tels Russell, Wittgenstein, Ramsey ou Reichenbach, ce qui détonne dans le Québec francophone d'alors. De plus, il est au fait des résultats scientifiques de l'époque, qu'on parle physique quantique, astrophysique ou encore théorie de l'évolution, ce qui est tout à son honneur. Ses textes présentent en outre d'évidentes qualités formelles: cohérence et rigueur, subtilité, argumentations serrées. Constituent-ils pour autant une philosophie pertinente aujourd'hui ? On peut en douter pour au moins deux raisons de taille. Tout d'abord, De Koninck accepte sans problème le fait que certains textes du stagirite soient périmés. Ainsi, dit-il, la physique et la chimie modernes ont rendu caducs le De Caelo et le De Generatione et Corruptione (156). Cependant, ajoute-t-il dans une formule qui laisse songeur, une bonne partie de sa Physique est, malgré tout, "à l'abri du temps ». On se demande bien au nom de quoi. Par ailleurs, l'allégeance ultramontaine imprime profondément sa marque sur ses écrits, ce qui les rend trop souvent dogmatiques, un trait habituel des néoscolastiques. À sa décharge, notons cependant que deux qualités tempèrent son orthodoxie. D'abord, il examine longuement la méthode appropriée aux divers sujets qu'il aborde. Cette approche, inspirée de la Critériologie apologétique du cardinal Mercier, était devenue usuelle dans l'univers néothomiste, mais notre philosophe en fait un usage original. Ensuite, un accent particulier colore ses écrits. Il assortit en effet ses analyses de commentaires pénétrants de saint Thomas et d'Aristote, qu'il possède sur le bout des doigts, et dont il cite constamment les textes, soit en latin avec traduction pour le premier, soit en français pour le second. (Apparemment, il était moins à l'aise avec le grec qu'avec le latin.) Pourquoi revenir ainsi aux écrits originaux? C'est que "l'argument d'autorité est en philosophie le plus débile» (292). Notons d'ailleurs que, souvent, il utilise les gloses perspicaces de saint Thomas pour éclairer des passages d'Aristote. Alors, thomisme ou aristotélisme? Ni l'un ni l'autre et... les deux à la fois. Qu'est-ce à dire ? Sans verser dans la casuistique, on constate que, pour lui, la théologie thomiste corrige et accomplit Aristote. Tandis que du point de vue métaphysique, le thomisme représente seulement une interprétation privilégiée de l'aristotélisme.

Cela dit, De Koninck a cru sincèrement à la pertinence de ces deux penseurs pour la modernité. Malgré quelques fulgurances passagères, les neuf écrits de ce volume, assez disparates, ne convaincront guère le lecteur postmoderne du bien-fondé d'une telle thèse.

En terminant, il faut mentionner un sérieux irritant. Les coquilles pullulent dans certains textes du volume, au point de gêner parfois la lecture. Pour amorcer une entreprise d'une telle importance, c'est vraiment regrettable, et ce handicap risque de limiter la diffusion internationale de l'ouvrage. Par ailleurs, les deux traductions inédites sont malheureusement grevées de maladresses et, il faut bien l'avouer, de navrantes fautes de gram- 
maire. Espérons que les volumes à venir ne souffriront pas des mêmes tares et qu'on apportera les correctifs nécessaires. Cela s'impose d'autant plus que De Koninck a constitué, au Québec, un relais cardinal. C'est pourquoi, plus qu'un simple matériau à recenser, ses écrits constituent d'authentiques documents. Telles ces pierres milliaires, patinées par le temps ou cachées par la végétation, ils indiquent un chemin autrefois fréquenté. S'il a été abandonné depuis, si la modernité philosophique a frayé d'autres voies, l'historien des idées trouvera avantage à l'arpenter à nouveau. Car, comme le disait Fernand Dumont de notre passé intellectuel: «Tant que les écrits de Dessaulles ou de Laflèche nous apparaîtront comme des niaiseries, nous n'aurons rien compris. En cette matière, on se saisit que ce dont on a transgressé l'étrangeté, on n'appréhende que ce dont on s'est fait soi-même le sujet ${ }^{5}$.» L'orthodoxie néothomiste a puissamment marqué l'histoire du Québec. Ne serait-ce qu'à ce titre, elle doit s'inscrire, fût-ce à notre corps défendant, dans la mémoire collective.

JEAN-CLAUDE SIMARD

Collège de Rimouski

Tilly, Charles. Explaining Social Processes, Boulder et Londres, Paradigm Publishers, 2008, $215 \mathrm{p}$.

À sa mort en 2008, le sociologue et historien américain Charles Tilly laissait derrière lui une œuvre d'une cinquantaine d'ouvrages. Docteur de Harvard en 1958, Tilly entama sa carrière à une époque où la sociologie américaine était dominée par l'influence de Talcott Parsons. Il prit rapidement ses distances avec ce dernier. Comme la plupart de ceux qui rejetèrent la sociologie parsonienne, il en dénonçait la pauvreté historique, l'absence d'une perspective rigoureusement comparative et l'incapacité à rendre compte du changement social au-delà de l'a priori téléologique de la théorie de la modernisation. Bien que certaines contributions de Tilly appartiennent clairement au registre de l'histoire sociale (1964), c'est avec ses contributions à l'étude des répertoires de conflits politiques et à celle de la sociologie historique que Tilly laissa son empreinte sur les sciences sociales américaines. Depuis Parsons, peu de sociologues ont eu une telle influence sur le développement de la sociologie américaine.

Le nom de Tilly évoque davantage la discipline historique et sociologique que la philosophie ou la philosophie des sciences sociales. Dans ses écrits, la réflexion épistémologique est certainement moins omniprésente que chez un sociologue comme Pierre Bourdieu. Pourtant, les philosophes

5. «Le projet d'une histoire de la pensée québécoise », Le sort de la culture, Montréal, l'Hexagone, 1995, p. 364; Dumont souligne. 